\section{The disutility of the hard-easy effect in choice confidence}

\author{
Edgar C. MerkLe \\ Wichita State University, Wichita, Kansas
}

A common finding in confidence research is the hard-easy effect, in which judges exhibit greater overconfidence for more difficult sets of questions. Many explanations have been advanced for the hard-easy effect, including systematic cognitive mechanisms, experimenter bias, random error, and statistical artifact. In this article, I mathematically derive necessary and sufficient conditions for observing a hard-easy effect, and I relate these conditions to previous explanations for the effect. I conclude that all types of judges exhibit the hard-easy effect in almost all realistic situations. Thus, the effect's presence cannot be used to distinguish between judges or to draw support for specific models of confidence elicitation.

Confidence and its calibration are oft-studied topics in the decision sciences. The topics are relevant to many applied areas, including finance (Thomson, Önkal-Atay, Pollock, \& Macaulay, 2003), meteorology (Murphy \& Winkler, 1984), and eyewitness testimony (Wells, Ferguson, \& Lindsay, 1981). Psychological research on confidence also has implications for the elicitation of prior distributions in Bayesian models (e.g., O'Hagan et al., 2006). This general applicability of confidence elicitation contributes to its popularity as a research area.

In the applications above, confidence is usually expressed as a probability: Given a single event, 0 expresses certainty that the event will not occur, 1 expresses certainty that the event will occur, and intermediate probabilities express intermediate levels of certainty. This is known to decision researchers as a no choice-100 (NC100) task (terminology from Ronis \& Yates, 1987). In an alternative task, the choice-50 (C50) task, judges choose between two alternatives and then report confidence in their choice. Confidence is bounded at .5 and 1 because, if the judge's confidence is below .5 , he or she should have chosen the other alternative.

Regardless of the task, researchers often examine a judge's calibration by comparing average confidence $(\bar{f})$ over a set of events to proportion correct $(\bar{d})$ over the same set. This results in the overconfidence statistic (OC):

$$
\mathrm{OC}=\bar{f}-\bar{d} .
$$

Judges are said to be well calibrated if $\mathrm{OC}=0$ - that is, if their average confidence matches proportion correct. It is very common to find that $\mathrm{OC}>0$ - that is, that judges are overconfident.
A second ubiquitous finding in confidence research deals with the magnitude of OC at different difficulty levels. This finding, termed the hard-easy effect, was described in detail by Lichtenstein, Fischhoff, and Phillips (1982; see also Lichtenstein \& Fischhoff, 1977). They found that people tend to exhibit more overconfidence for hard sets of questions than for easy sets of questions. Across experiments or question sets, a hard-easy effect for the C50 task is displayed in Figure 1. Proportion correct is on the $x$-axis, overconfidence is on the $y$-axis, and each point represents a hypothetical experiment or question set. The points show the general hard-easy trend: As $\bar{d}$ increases, OC decreases.

Many explanations have been advanced for the hardeasy effect, including insufficient placement of confidence criteria in a signal detection framework (Ferrell \& McGoey, 1980; Suantak, Bolger, \& Ferrell, 1996), random error (Erev, Wallsten, \& Budescu, 1994), the insensitivity of confidence to task difficulty (Price, 1998; von Winterfeldt \& Edwards, 1986), and cognitive bias (Griffin \& Tversky, 1992). Whereas these explanations refer to within-judge factors, other researchers have proposed that the experimental design itself contributes to the hard-easy effect. For example, Gigerenzer, Hoffrage, and Kleinbölting (1991) showed that the biased selection of test questions can yield a hard-easy effect: If an experimenter chooses more trick questions than are usually found in some domain, for example, we might expect a judge's confidence to be artificially high and accuracy to be artificially low.

Juslin, Winman, and Olsson (2000) quantified the hard-easy effect as a regression weight between $\bar{d}$ and OC. In this article, I expand upon this insight and study the covariance between proportion correct and overconfidence. If this covariance is negative, a hard-easy effect is observed: As proportion correct increases, overconfidence tends to decrease. In deriving conditions under which the covariance is negative, I derive necessary and sufficient conditions for observing a hard-easy effect in empirical data. These conditions provide a unifying framework for comparing and contrasting the explanations for the hardeasy effect described above, as well as for determining the extent to which these explanations are empirically separable.

With the analyses in this article, I formalize and extend a number of other researchers' verbal arguments and simple demonstrations. Wallsten (1996) argued that confidence researchers had placed too much emphasis on calibration and too little emphasis on cognitive processes. He showed the extent to which different analyses could impact the experimental results (see also Dawes \& Mulford, 1996; Erev et al., 1994), and he demonstrated how the hard-easy effect could be a symptom of the test (rather

E. C. Merkle, edgar.merkle@wichita.edu 




Figure 1. Graph of the hard-easy effect. Each point on the graph represents a hypothetical experimental outcome, and the negative trend of the points represents the hard-easy effect. The two points in the gray box demonstrate a reversal of the hardeasy effect.

than of the judge). In this article, I am not concerned with specific explanations for the hard-easy effect; I instead argue that the effect cannot help us distinguish among the potential explanations. To be specific, I mathematically derive conditions that are necessary and sufficient for observing a hard-easy effect. In relating these conditions to a general model of judges, I show that the hard-easy effect will occur in almost all experiments. Thus, presence of the effect tells us nothing about the confidence elicitation process.

In the following pages, I first derive necessary and sufficient conditions for observing the hard-easy effect. Next, I use an error model of confidence to examine situations in which the sufficient condition is satisfied. The situations include both $\mathrm{C} 50$ and $\mathrm{NC} 100$ experiments, described at the beginning of the article. Finally, I discuss the general implications of my analyses for confidence research and modeling.

\section{NECESSARY AND SUFFICIENT CONDITIONS FOR A HARD-EASY EFFECT}

As described in the introduction, the hard-easy effect can be viewed as a description of the covariance between proportion correct and overconfidence $[\operatorname{cov}(\bar{d}, \mathrm{OC})]$ : Experimental findings show that a negative relationship tends to exist between these two measures. This relationship is shown in Figure 1, which is a scatterplot of $\bar{d}$ versus OC. Each point represents the $\bar{d}$ and OC calculated for a single test, and the negative trend of the points represents a hard-easy effect. The dotted line is the regression line for these data.

Following Figure 1, my analyses focus on hard-easy effects at the test level (i.e., where each point in the graph represents a single test). Although I focus on a single judge completing a series of tests, the number of judges who take the tests is unimportant: We could plot a single judge's data across a series of tests as well as a group's data across a series of tests. I ignore within-test analyses, in which accuracy is calculated within different confidence bins and calibration curves are employed (see, e.g., Dougherty, 2001; Ferrell \& McGoey, 1980; Lichtenstein et al., 1982; Merkle \& Van Zandt, 2006), because these analyses do not immediately take into account the proportion of responses within each confidence bin, which can lead to misleading results (see, e.g., Wallsten, 1996).

To examine the ubiquity of the hard-easy effect, we can expand $\operatorname{cov}(\bar{d}$, OC $)$. In the equations below, $\bar{d}$ is proportion correct and $\bar{f}$ is average confidence at the test level. Following much empirical decision-making research, I assume that the $f_{i}$ values are scaled from 0 to 1 (or, for $\mathrm{C} 50$ tasks, from .5 to 1 ).

$$
\begin{aligned}
\operatorname{cov}(\bar{d}, \text { OC }) & =\operatorname{cov}(\bar{d}, \bar{f}-\bar{d}) \\
& =\operatorname{cov}(\bar{d}, \bar{f})-\operatorname{var}(\bar{d}) \\
& =\operatorname{sd}(\bar{d}) \operatorname{sd}(\bar{f}) \operatorname{corr}(\bar{d}, \bar{f})-\operatorname{var}(\bar{d}) \\
& =\operatorname{sd}(\bar{d})[\operatorname{sd}(\bar{f}) \operatorname{corr}(\bar{d}, \bar{f})-\operatorname{sd}(\bar{d})] .
\end{aligned}
$$

Given Equation 2, it is possible to derive necessary and sufficient conditions for the hard-easy effect.

Proposition 1. Assume that the hard-easy effect is defined across tests as a negative covariance between $\bar{d}$ and $O C$. Then (A) we will observe a hard-easy effect if and only if $s d(\bar{f}) \operatorname{corr}(\bar{d}, \bar{f})<s d(\bar{d})$ (necessary condition), and (B) we will observe a hard-easy effect if $\operatorname{var}(\bar{f})<\operatorname{var}(\bar{d})$ (sufficient condition).

Proof. (A) In Equation 2, all standard deviations are greater than or equal to 0 . Thus, the sign of Equation 2 depends entirely on the difference in brackets. (B) By definition, $\operatorname{corr}(\bar{d}, \bar{f}) \leq 1$. Thus, $\operatorname{sd}(\bar{f}) \operatorname{corr}(\bar{d}, \bar{f}) \leq$ $\operatorname{sd}(\bar{f})$. The sufficient condition (expressed in Proposition 1 as variances instead of as standard deviations) automatically satisfies the necessary condition.

An immediate implication from Proposition 1A (the necessary condition) is that judges who report confidence judgments that are completely unrelated to the stimulus will always exhibit a hard-easy effect. In such a case, $\operatorname{corr}(\bar{d}, \bar{f})=0$ [and $\operatorname{sd}(\bar{d})>0$ for realistic tests]. Thus, through nothing interesting on his or her part, the occasional undergraduate who completes a 45-min confidence experiment in 3 min will exhibit a hard-easy effect. An interesting aspect of Proposition 1B (the sufficient condition) is that it contains a definition of insensitivity to task difficulty: Confidence is not affected by task difficulty as much as it should be, and, in turn, mean confidence varies less than does proportion correct. I will return to this issue in the General Discussion section.

Judges (and mathematical models of confidence) whose confidence and choice satisfy the Proposition 1B condition will exhibit a hard-easy effect. Thus, it is of interest to determine the ease with which this sufficient condition 
is satisfied. If every judge satisfies the condition on every test, it is useless to seek a scientific explanation for it. That is, if a hard-easy effect is always present, a model's ability to exhibit the effect is meaningless.

Upon initial examination, it appears that Proposition 1B is not very stringent. In reviewing sets of confidence experiments, some researchers have noted that the variance of accuracy tends to be larger than that of confidence (Dawes \& Mulford, 1996; Juslin et al., 2000). Consider further a C50 task, in which judges choose one of two alternatives and then give a confidence rating for their chosen alternative. ${ }^{1}$ In this task, confidence ranges from .5 to 1 , because the judge should choose the alternative that he or she believes is more likely to be correct (i.e., if confidence in the chosen alternative is below .5, the judge should have chosen the other alternative). As a result, $\bar{d}$ is based on a series of $0 \mathrm{~s}$ and $1 \mathrm{~s}$, whereas $\bar{f}$ is based on numbers that range from .5 to 1 . Within any one experiment, this leads us to surmise that the variance of accuracy would tend to be greater than the variance of confidence. The greater within-experiment variance of accuracy might naturally lead to greater between-experiment variance. If this is the case, the condition in Proposition 1B is satisfied. We thus observe a hard-easy effect, regardless of the psychological mechanisms underlying confidence elicitation.

Although the arguments above are intuitive, they are not formal enough to allow us to draw any definitive conclusions. In the next section, I mathematically develop the arguments to show that the condition in Proposition 1B is satisfied in almost all realistic situations.

\section{SATISFYING THE SUFFICIENT CONDITION}

To study when the condition in Proposition 1B is satisfied, I use an error model of confidence ${ }^{2}$ (Juslin, Olsson, \& Björkman, 1997) that resembles realistic judges in two-alternative confidence experiments. For each test item, the model assumes that judges have well-calibrated, internal confidence in the correctness of each alternative. Choice is based on these internal confidence judgments, so that the judge chooses the alternative that is more likely to be correct. Random error then enters into the translation from internal confidence to reported confidence. For a judge responding to item $k(k=1, \ldots, K)$ on test $j(j=$ $1, \ldots, J)$, this is expressed as

$$
f_{j k}=t_{j k}+e_{j k},
$$

where $f_{j k}$ is reported confidence (bounded between 0 and 1 ), $t_{j k}$ is internal confidence (bounded between 0 and 1), and $e_{j k} \sim \mathrm{N}\left(0, \sigma^{2}\right)$ (unbounded $\left.{ }^{3}\right) . t_{j k}$ also reflects test difficulty, because we assume that these internal confidence judgments are perfectly calibrated.

The model above is very general; Merkle, Sieck, and Van Zandt (2008) showed that the error term in this model can account for a number of systematic biases. Thus, I can use the model to generally examine the frequency with which the hard-easy effect occurs. I make no arguments that the error model best describes the confidence elicita- tion process; I simply use the error term to encompass many potential explanations for the hard-easy effect.

Assume that a single judge completes $J$ tests, which have $K$ items each. Further assume that item difficulty (described by the $t \mathrm{~s}$ ) arises from the same distribution across tests. If $K=\infty$ (infinite number of items per test), each test would have the same difficulty. However, given the relatively small values of $K$ in many experiments, we can obtain tests of varying difficulty while sampling all ts from the same distribution. This is a simplifying assumption that could be relaxed if necessary.

Given a specific distribution of internal confidence judgments $(t \mathrm{~s})$ for the tests and a constant error variance, the error model's predictions for $\operatorname{var}(\bar{f})$ and $\operatorname{var}(\bar{d})$ are given by Equations 4 and 5, respectively (see Appendix A for more details; $E$ is the expectation operator).

$$
\begin{aligned}
& \operatorname{var}(\bar{d})=\frac{1}{J K}\{E[t(1-t)]+\operatorname{var}(t)\} \\
& \operatorname{var}(\bar{f})=\frac{1}{J K}\left[\operatorname{var}(t)+\sigma^{2}\right] .
\end{aligned}
$$

For a specific experimental paradigm, these equations represent the error model's predictions of how $\bar{d}$ and $\bar{f}$ vary across tests. If the variance in Equation 4 is larger than that of Equation 5, we will observe a hard-easy effect. Substituting these equations into Proposition 1B and rearranging, we will observe a hard-easy effect if

$$
\sigma^{2}<E[t(1-t)]
$$

An immediate implication from Equation 6 is that, when judges give perfectly calibrated confidence judgments at the item level, they will almost always exhibit a hard-easy effect at the test level. Perfectly calibrated at the item level means that, for each test item, the judge reports a confidence judgment that equals his or her probability of choosing the correct answer. In other words, when a judge reports confidence of $c$ for some item, he or she chooses the correct answer $c \%$ of the time. Test level means that we give a judge multiple tests and calculate the average proportion correct and overconfidence for each test. In the language of the error model (Equation 3), perfect calibration at the item level means that $\sigma^{2}=0$. Furthermore, regardless of the specific tests that a judge takes, $E[t(1-\mathrm{t})] \geq 0 . .^{4}$ Thus, Equation 6 is almost always satisfied.

The fact that perfect calibration at the item level leads to a hard-easy effect at the test level is unintuitive. Perfect calibration is usually defined at the test level, where, referring to Figure 1, perfectly calibrated judges would follow the horizontal line along $\mathrm{OC}=0$. If judges are perfectly calibrated at the item level, however, they can exhibit a hard-easy effect due to the Bernoulli error $(\varepsilon)$ inherent in $\bar{d}$. In this situation, we can write $\bar{d}=\bar{f}+\varepsilon$ and $\mathrm{OC}=\bar{f}-\bar{d}=-\varepsilon$. Positive or negative values of $\varepsilon$ make $\bar{d}$ large or small, respectively, and OC small or large, respectively. This results in a hard-easy effect. ${ }^{5}$

The findings thus far go a long way toward rendering the hard-easy effect an uninteresting phenomenon: The effect is present for both perfect judges and awful judges. I proceed, however, to examine the ubiquity of 
the hard-easy effect when judges are neither perfect nor awful. This is more similar to real judges in real confidence experiments.

\section{Example 1: C50 Task}

I first examine the relative magnitudes of $\operatorname{var}(\bar{d})$ and $\operatorname{var}(\bar{f})$ in the context of C50 tasks, where judges first choose one of two alternatives and then report confidence ranging from .5 to 1 . For these tasks, an initial distribution from which we may elect to sample the $t_{j k}$ is the Uniform $(.5,1)$. With this distribution, it can be shown that $E[t(1-t)]=.167$. Therefore, to observe a hard-easy effect, the error variance must be less than $.167 .{ }^{6}$ In the context of real experiments, the .167 error variance is massive: Across many contexts, Juslin et al. (2000; Juslin, Winman, \& Olsson, 2003) estimated $\sigma^{2}$ to be between .015 and .03 . Merkle et al. (2008) and Olsson, Juslin, and Winman (2008) both discuss the fact that this estimate is surprisingly large. These estimates are nowhere close to .167, indicating that, if judges respond in a C50 task according to the error model, they will always exhibit the hard-easy effect.

A major assumption underlying the example above was the distribution of the $t_{j k}$. Whereas I assumed a Uniform $(.5,1)$ distribution, a more realistic distribution might involve many of the $t_{j k} \mathrm{~s}$ being clustered around .5 or 1 (see, e.g., Erev et al., 1994; Wallsten, 1996). For simplicity, I specify here a discrete distribution of $t_{j k}$ based on that from Erev et al.:

$$
P\left(T_{j k}=t_{j k}\right)=\left\{\begin{array}{l}
.3 \text { for } t_{j k}=.50 . \\
.1 \text { for } t_{j k}=\{.60, .70, .80, .90\} . \\
.3 \text { for } t_{j k}=1 . \\
0 \text { otherwise. }
\end{array}\right.
$$

Under this distribution, $E[t(1-t)]=.145$. Although this expectation is smaller than that of the Uniform distribution, it is still much larger than the $\sigma^{2}$ estimates from previous research. This means that we would still observe a hardeasy effect when the $t_{j k} \mathrm{~s}$ arise from the U-shaped distribution. In the next example, I show that these results extend to tasks in which judges use a $0-1$ confidence scale.

\section{Example 2: NC100 Task}

Whereas the derivations above demonstrate the ubiquity of the hard-easy effect in C50 tasks, the situation does not obviously extend to NC100 tasks. In these tasks, judges do not choose between two alternatives. Instead, they simply give a $0-1$ confidence judgment as to the truth of one specific alternative. Wallsten (1996) discussed the fact that NC100 tasks are preferable to C50 tasks because the base rate of true items is entirely in the experimenter's control. In contrast, the base rate of correct items in C50 tasks depends both on the experimenter's selection of test questions and on the judge's knowledge of those questions. This can enhance any hard-easy effect that may already have been present.

Sampling $t_{j k}$ from a Uniform $(0,1)$ distribution, we can calculate $E[t(1-t)]=.167$. This expectation is the same as that of the Uniform $(.5,1)$ distribution from the C50 task. Thus, the same arguments apply: To make the hardeasy effect disappear, we must have $\sigma^{2} \geq .167$. Given that this variance is unrealistic in empirical data (Juslin et al., 2000; Merkle et al., 2008), judges will always yield a hard-easy effect.

Similar to the U-shaped distribution in the C50 task, a $\mathrm{W}$-shaped distribution is more realistic for $t_{j k}$ in an $\mathrm{NC} 100$ task. Such a distribution indicates a preponderance of items that participants know are true, know are false, or know nothing about. Whereas Erev et al. (1994) employed a discrete W-shaped distribution on the $t_{j k}$, I use here a mixture of independent Beta distributions:

$$
f(t) \sim \frac{1}{2}[\operatorname{Beta}(20,20)+\operatorname{Beta}(.25, .25)] .
$$

The Beta distribution is characterized by two parameters (often labeled $\alpha$ and $\beta$ ), and it is bounded at 0 and 1 . When $\alpha=\beta$, as is the case for both Beta distributions above, the distribution is symmetric around .5. The mixture distribution above roughly corresponds to the discrete distribution used by Erev et al., and it also lifts the restriction that judges' internal probabilities must be multiples of .10. The distribution is displayed in Figure 2.

The Beta mixture distribution of $t_{j k}$ s yields $E[t(1-t)]=$ .207. This is again larger than realistic $\sigma^{2}$ values, and it is also larger than the figure derived for the Uniform distribution above. This continues the general trend of observing hard-easy effects in all realistic situations.

In an effort to find $t_{j k}$ distributions that do not result in a hard-easy effect, I explored asymmetric Beta distributions (those for which $\alpha \neq \beta$ ). For example, consider the $\operatorname{Beta}(.25,1)$ distribution: This reflects an NC100 test in which participants know that most items are false (see Figure 3 ). To be specific, $56 \%$ of the true probabilities ( $t$ s) lie below .1. Although this distribution is considerably different from the others that $\mathrm{I}$ considered, it still yields $E[t(1-t)]=$

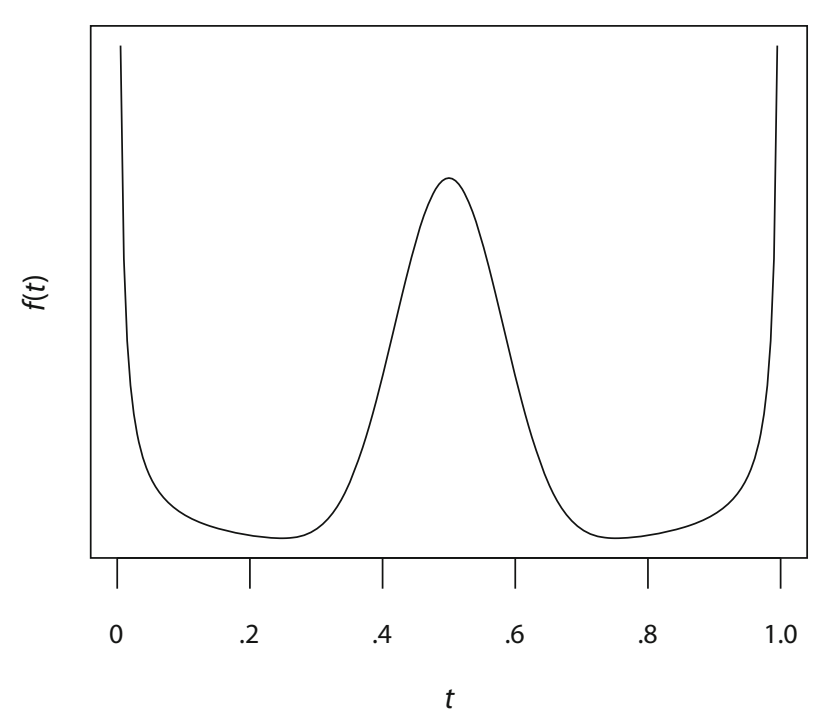

Figure 2. Mixture of $\operatorname{Beta}(20,20)$ and $\operatorname{Beta}(.25, .25)$ densities. 


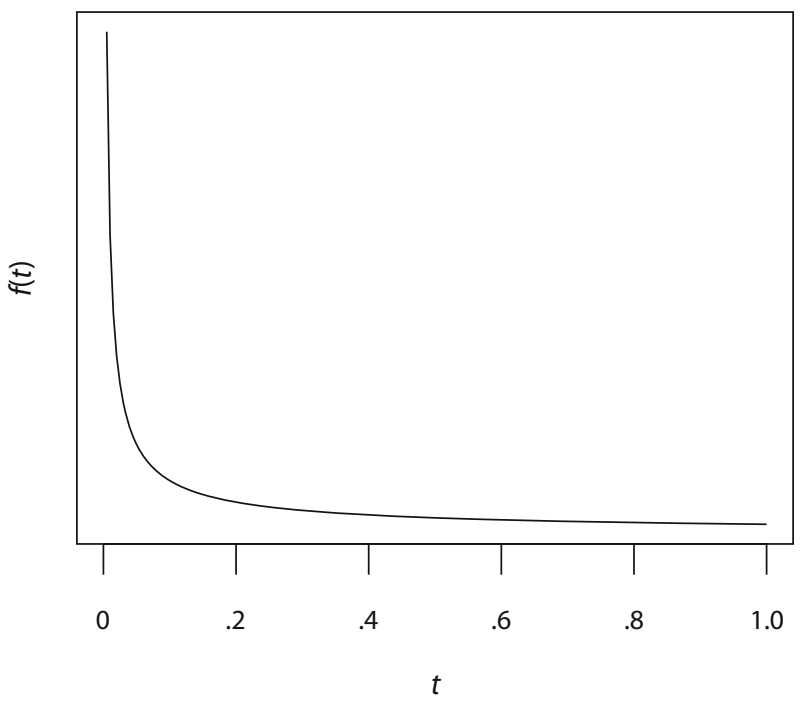

Figure 3. The Beta $(.25,1)$ density.

.089. This is still far from the $.015-.03 \sigma^{2}$ range that Juslin et al. $(2000,2003)$ estimated from previous data.

\section{Making the Hard-Easy Effect Disappear}

Are there specific types of tests that make the hardeasy effect disappear for realistic judges? In other words, what values of $\alpha$ and $\beta$ can we take so that $E[t(1-t)] \leq$ .03 ? To answer this question, I derive an expression for $E[t(1-t)]$ as a function of the Beta distribution parameters $\alpha$ and $\beta$ :

$$
E[t(1-t)]=\frac{\alpha^{2} \beta+\alpha \beta^{2}}{(\alpha+\beta)^{2}(\alpha+\beta+1)} .
$$

I then insert this expression into Equation 6, resulting in the following condition for observing a hard-easy effect:

$$
\sigma^{2}<\frac{\alpha^{2} \beta+\alpha \beta^{2}}{(\alpha+\beta)^{2}(\alpha+\beta+1)} .
$$

Three-dimensional contour plots of Equation 8 appear in Figure 4 as a function of $\alpha$ and $\beta$ (in the plots, $E$ stands for $E[t(1-t)])$. Both plots display the same contour, but from different angles. For different values of $\alpha$ and $\beta$, the plots show values of $\sigma^{2}$ that judges must achieve to make the hard-easy effect disappear. In other words, we can horizontally slice the plots at $E=.03$. Whenever the slicing touches the contour, we have found a test (values of $\alpha$ and $\beta$ ) for which realistic judges do not exhibit a hardeasy effect.

These plots show that, to obtain $E[t(1-t)] \leq .03$, we need either $\alpha$ or $\beta$ to be very close to 0 . When $\alpha$ is close to 0 , the majority of the $t_{j k}$ density is close to 0 . When $\beta$ is close to 0 , the majority of the $t_{j k}$ density is close to 1 . These reflect situations in which judges are certain that every item is false $(\alpha \approx 0)$, certain that every item is true $(\beta \approx 0)$, or certain that some items are true and other items are false $(\alpha \approx \beta \approx 0)$. To summarize, the hard-easy effect will disappear only when judges are nearly certain about every question on a test. These types of tests are useless in confidence elicitation experiments: If judges know that every item is either true or false, confidence judgments add no extra diagnostic information.

Although the Beta distribution can generally account for tests of varying difficulties, it cannot immediately account for multimodal distributions. For example, the W-shaped distribution that I employed in Example 2 cannot arise from a single Beta distribution. To obtain the W-shaped distribution, I used a mixture of two symmetric Beta distributions. Under the constraints that $\alpha_{1}=\beta_{1}$ and $\alpha_{2}=\beta_{2}$, this mixture has the form
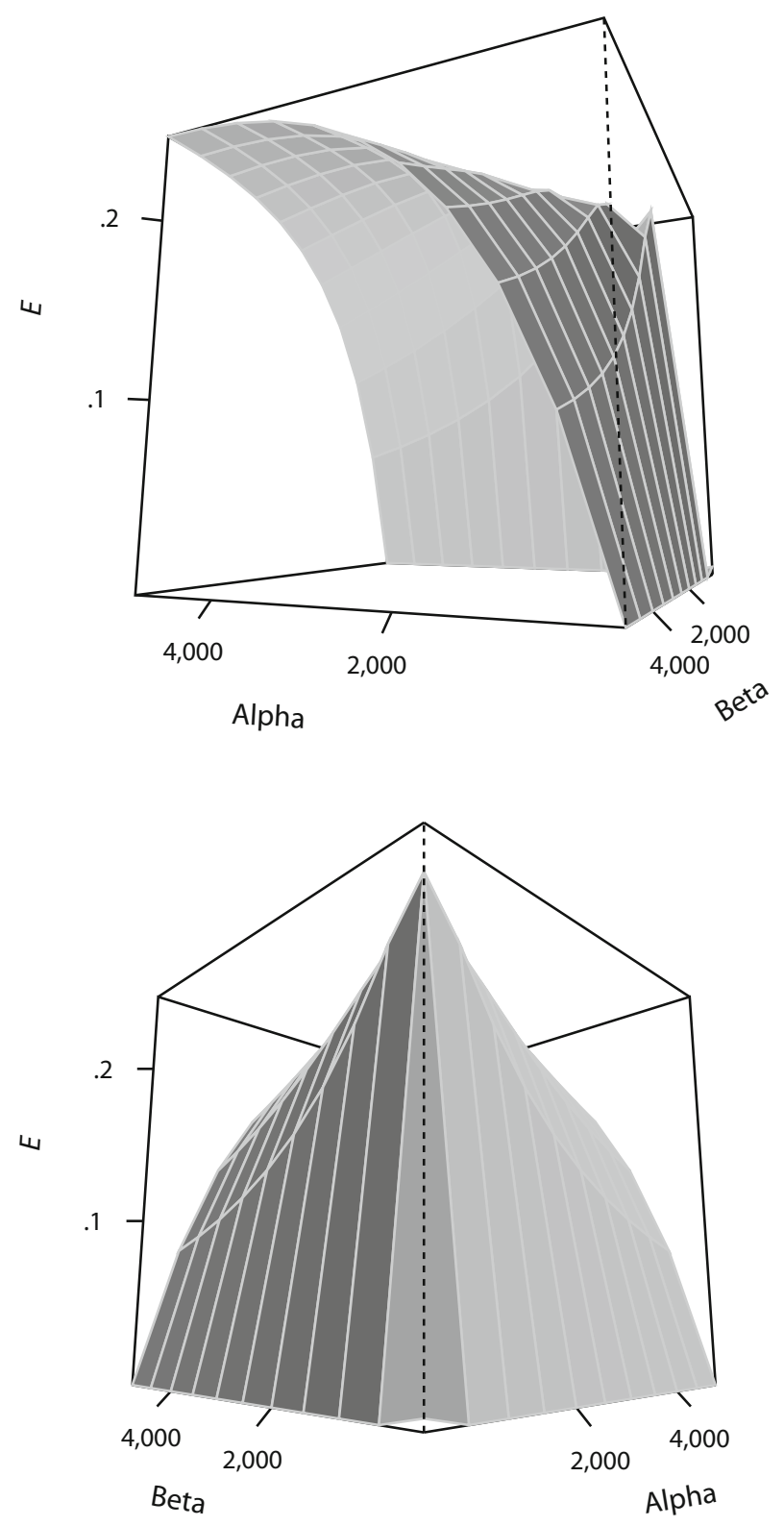

Figure 4. Contour plots of Equation 8 as a function of $\alpha$ and $\beta$. For the term on the right side of Equation 8 to be below .03, it is necessary that $\alpha \approx 0$ or $\beta \approx 0$. 


$$
f(t) \sim \frac{1}{2}\left[\operatorname{Beta}\left(\alpha_{1}, \beta_{1}\right)+\operatorname{Beta}\left(\alpha_{2}, \beta_{2}\right)\right] .
$$

For such mixtures of symmetric Beta distributions, it is possible to show that

$$
E[t(1-t)]=\frac{1}{4}\left[1-\frac{1}{4}\left(\frac{1}{\left(2 \alpha_{1}+1\right)}+\frac{1}{\left(2 \alpha_{2}+1\right)}\right)\right]
$$

Thus, we observe a hard-easy effect if

$$
\sigma^{2}<\frac{1}{4}\left[1-\frac{1}{4}\left(\frac{1}{\left(2 \alpha_{1}+1\right)}+\frac{1}{\left(2 \alpha_{2}+1\right)}\right)\right] .
$$
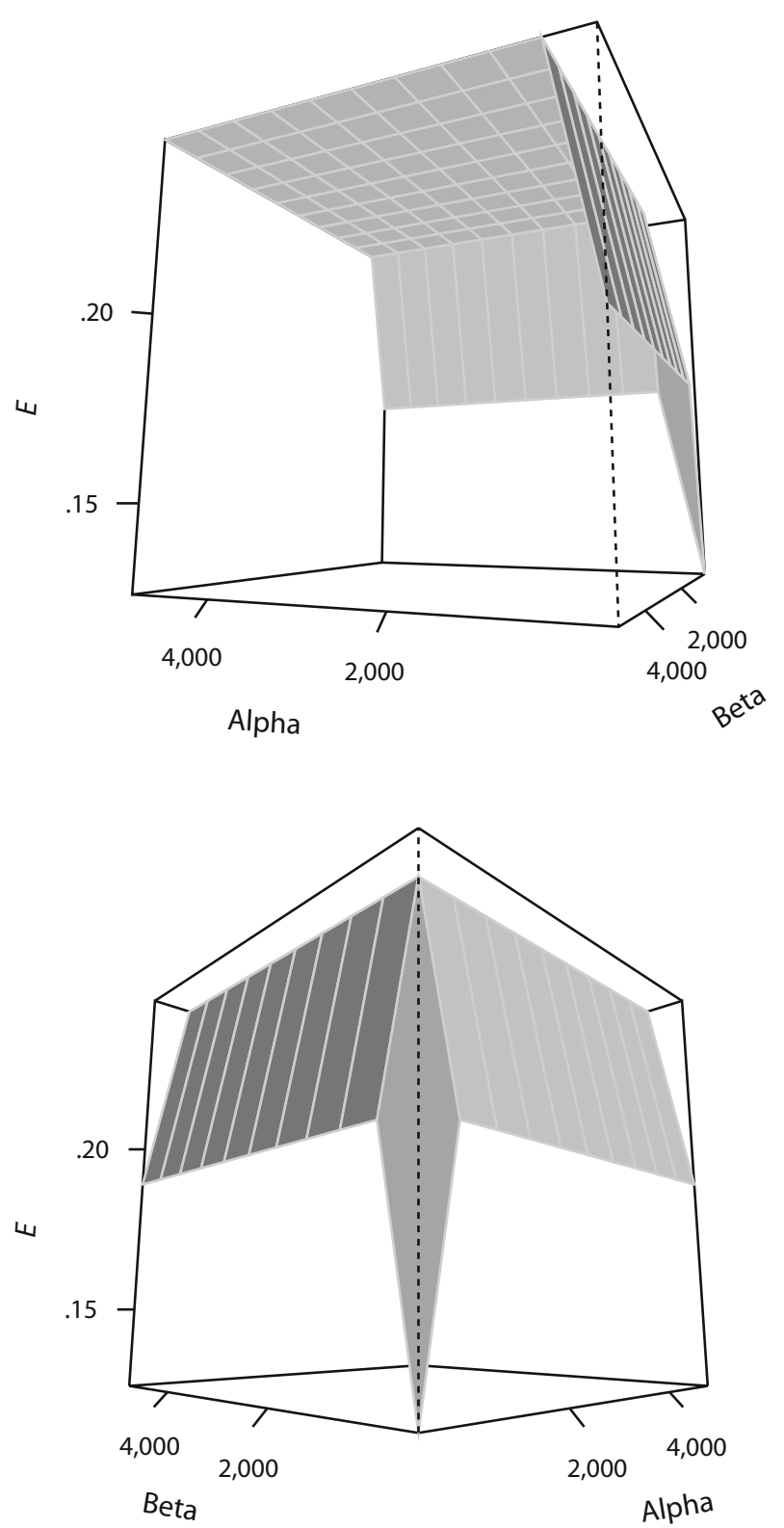

Figure 5. Contour plots of Equation 11 as a function of $\alpha$ and $\beta$. For the term on the right side of Equation 11 to be below .03, it is necessary that $\alpha \approx \beta \approx 0$.
Three-dimensional contour plots of Equation 11 appear in Figure 5 as a function of $\alpha$ and $\beta$ (in the plots, $E$ stands for $E[t(1-t)])$. Both plots display the same contour, but from different angles. Using the estimate of $E=.03$ to resemble realistic judges, we see that there exist almost no tests for which the hard-easy effect disappears. The only tests that do make the hard-easy effect disappear are those for which $\alpha \approx \beta \approx 0$. As discussed earlier, these are unrealistic tests for which judges are certain of all items. Thus, judges exhibit a hard-easy effect for all realistic tests that arise from a mixture of symmetric Beta distributions.

\section{Discussion}

The analyses above show that, when realistic judges respond in a confidence experiment according to the response-error model, the sufficient condition in Proposition 1 is satisfied in all realistic situations. This means that judges always exhibit a hard-easy effect in these situations. Wallsten (1996) addressed this topic in the context of a single $t_{j k}$ : He demonstrated that, given a constant response strategy in a C50 task, judges can be overconfident or underconfident, depending on the magnitude of $t_{j k}$ (leading to a hard-easy effect). The present derivations extend Wallsten's work by considering sets of $t_{j k}$ and responses that differ for each $t_{j k}$. They show the extent to which the hardeasy effect is ubiquitous in both $\mathrm{C} 50$ and NC100 tasks. In addition, the mathematical derivations show that perfectly calibrated judges (those for whom $\sigma^{2}=0$ ) and awful judges (those whose confidence judgments are unrelated to the stimulus) always exhibit the hard-easy effect.

\section{GENERAL DISCUSSION}

In this article, I have derived necessary and sufficient conditions for observing a hard-easy effect. Using a simple error model of confidence, I have examined situations under which we could expect to observe a hardeasy effect. Across both C50 tasks and NC100 tasks, I show that judges almost always exhibit hard-easy effects, even when their confidence judgments are perfectly calibrated at the item level. This result occurs in both C50 and NC100 tasks, and it is robust to changes in the distributions of internal probabilities $\left(t_{j k} \mathrm{~s}\right)$. Hard-easy effects were exhibited across uniform distributions of internal probabilities, W-shaped mixtures of Beta distributions, and any single Beta distribution reflecting a realistic test. In the following paragraphs, I discuss the extent to which my assumptions may have influenced my results, as well as implications of my findings.

\section{Assumptions}

I have employed three main assumptions to derive the results in this article. They are: (1) Judges respond according to the response-error model. (2) The $t_{j k} \mathrm{~s}$ follow a Beta distribution. (3) Overt confidence judgments $(f s)$ are not bounded between 0 and 1 . Although I have already addressed some of these assumptions, I directly discuss the impact of each of these assumptions below. 
Use of the error model. A major assumption of my analyses is that the error model with $\sigma^{2} \approx .03$ adequately describes real judges. If this assumption is grossly incorrect, there might still exist some realistic situations in which the hard-easy effect is absent. There are two main ways in which this assumption can be violated: (1) $\sigma^{2}$ is actually much larger than .03, or (2) the error model is simply a poor description of the confidence elicitation process.

As briefly addressed earlier in this report, previous confidence data and analyses have provided evidence that the error model is reasonable. Merkle et al. (2008) showed that the model can mimic data from other confidence models, such as the decision variable partition model (Ferrell \& McGoey, 1980) and models that incorporate alternativeunderweighting biases (McKenzie, 1997). Furthermore, Merkle et al. showed that the $\sigma^{2}$ estimates from Juslin et al. (2000, which are similar to estimates from Björkman, 1994, and Juslin et al., 1997) likely incorporate systematic biases as well as error. Thus, the error model with $\sigma^{2}=.03$ yields data that are similar to empirical data (Juslin et al., 2000, reported an $R^{2}$ of .99), even though judges obviously do not respond according to the model. Given that the hard-easy effect is one of observed data and is not tied to a specific confidence elicitation process, this assumption seems reasonable.

Use of the Beta distribution. The second assumption is that judges' true probabilities $\left(t_{j k} \mathrm{~s}\right)$ arise from a Beta distribution (although I did examine a small number of other distributions). The Beta distribution is flexible, encompassing the Uniform $(0,1)$ distribution as well as many asymmetric distributions. Thus, many plausible $t_{j k}$ distributions can arise from the Beta distribution. The Beta distribution cannot, however, generally account for multimodal distributions. For example, the Beta cannot immediately yield a W-shaped distribution, in which judges know many test questions and are completely uncertain of others. I employed a mixture of symmetric Beta distributions to examine this situation, but I could not undertake an exhaustive analysis of all possible beta mixture distributions. Given the other analyses, however, I would be surprised to find a beta mixture distribution that both resembles a realistic test and fails to yield a hard-easy effect.

Unbounded confidence judgments. Finally, in comparing $\bar{f}$ with $\bar{d}$, I did not account for the fact that reported confidence judgments are bounded between 0 and 1 . The addition of random error to each $t_{j k}$ allows for the possibility that some confidence judgments could become smaller than 0 or larger than 1 . The sufficient condition that I employed for observing a hard-easy effect, however, is $\operatorname{var}(\bar{f})<\operatorname{var}(\bar{d})$. If I did account for the confidence bounds in my analyses, $\operatorname{var}(\bar{f})$ would decrease and $\operatorname{var}(\bar{d})$ would remain the same. Thus, the observation of a hardeasy effect in my analyses implies the observation of a hard-easy effect in bounded confidence data. Although it was mathematically simpler to disregard the bounds of the confidence scale, it also resulted in conservative analyses. Further evidence supporting this claim appears in Appendix B, where I describe simulations that explicitly account for the bounds of the confidence scale.

\section{Reversals of the Hard-Easy Effect}

Gigerenzer et al. (1991) discussed two studies (Keren, 1988; Ronis \& Yates, 1987) that yielded a total of four hard-easy effect reversals. There is nothing systematic about these two studies: Each was intended to study something other than the hard-easy effect, and the original authors provided few analyses that are specifically relevant to the hard-easy effect. However, within some conditions of both studies, participants exhibited more overconfidence for an easy test than for a hard test (see Gigerenzer et al., 1991, Figure 10). These hard-easy reversals initially appear to invalidate the present results: I have argued that the hardeasy effect always occurs (i.e., that the covariance between proportion correct and overconfidence is always negative), whereas Gigerenzer et al. discussed studies in which it did not occur (i.e., in which the covariance appeared positive).

Upon closer examination, the hard-easy effect definition employed in this article $[\operatorname{cov}(\bar{d}$, OC $)<0]$ can accommodate reversals of the sort described by Gigerenzer et al. (1991). An example of this is shown within the gray box in Figure 1. The two points within the box depict a hard-easy reversal: There is greater overconfidence for the easier test. Examining the overall trend of all the points in the graph, however, there is a clear hard-easy effect. In general, two tests are insufficient to reliably assess the covariance that defines the hard-easy effect.

\section{Verbal Versus Mathematical Definitions}

The sufficient conditions derived in this article can also be used to clarify explanations for overconfidence and the hard-easy effect. As described earlier, the sufficient condition for the hard-easy effect in Proposition 1 contains one definition of insensitivity to task difficulty. Thus, we might accept insensitivity to task difficulty as a valid explanation for the effect. This explanation agrees with those of many other researchers (e.g., Lindsay, Nilsen, \& Read, 2000; Merkle \& Van Zandt, 2006; Price, 1998; Sieck, Merkle, \& Van Zandt, 2007; Weber \& Brewer, 2004), who have discussed empirical findings related to insensitivity to task difficulty. Interestingly, however, Proposition 1 shows that insensitivity to task difficulty is not necessary for the hard-easy effect to occur.

Two further caveats can be made on the "insensitivity to task difficulty" explanation. First, the definition in Proposition 1B is data driven: We know whether a judge is insensitive to task difficulty by directly examining whether a set of data satisfy the condition in Proposition 1B. This makes insensitivity to task difficulty more of an effect than an explanation. Other explanations for overconfidence and the hard-easy effect are more process driven. For example, McKenzie (1997) argued that, in assessing confidence, judges "underweight the alternative": They focus on their chosen alternative and ignore the unchosen alternative(s). There is nothing in the observed data that tells us definitively whether alternative underweighting occurred; we must rely on experimental manipulations designed to impact alternative underweighting. This type of explanation for overconfidence and the hard-easy effect is potentially more useful than that in Proposition 1B. 
A second caveat is that we can define insensitivity to task difficulty in ways other than the definition in Proposition 1B. For example, Gigerenzer et al. (1991) defined the phrase as a judge's inability to accurately gauge his or her performance on a test. This definition, which does not necessarily involve confidence, led Gigerenzer et al. to conclude that their experiments "do not support the explanation of overconfidence and the hard-easy effect by assuming that subjects are insensitive to task difficulty" (p. 520). The authors specifically found that judges were relatively accurate in guessing their proportions correct across tests that varied in difficulty.

To summarize these experimental results, judges are relatively good at directly guessing their accuracy for a set of questions, and are relatively bad at indirectly guessing their accuracy via confidence judgments for each item. These results are consistent with the notion of separate psychological mechanisms for confidence and choice. ${ }^{7}$ Such a notion was discussed by Griffin and Tversky (1992), and it has recently been studied in the context of signal detection (Mueller \& Weidemann, 2008) and the assesssearch-construct model of confidence (Sieck et al., 2007). Furthermore, the paragraphs above demonstrate that we can define insensitivity to task difficulty in multiple ways and that these different definitions can lead us to different conclusions. This demonstration generally highlights the importance of mathematical definitions for psychological phenomena: Although verbal definitions are intuitive, they lack the precision to specifically define a phenomenon (see, e.g., Myung \& Pitt, 2001).

\section{Summary}

From the results in this article, I conclude that the hardeasy effect tells us nothing about the goodness of a judge or about the confidence elicitation process. From an empirical perspective, both perfectly calibrated judges (at the item level) and terrible judges (whose confidence is unrelated to the stimulus) exhibit the hard-easy effect. From a modeling perspective, the hard-easy effect occurs for all realistic judges and tests. Any model that provides a reasonable fit to observed data will exhibit the effect. Thus, the effect cannot help us learn about the confidence elicitation process, because it cannot help us discriminate between potential confidence elicitation models. These results formalize and extend Wallsten's (1996) statement that the hard-easy effect and overconfidence are "not suitable for investigating basic cognitive processes" (p. 225). Instead, other confidence measures, such as response distributions or base rates, are necessary for the study of confidence elicitation mechanisms.

\section{AUTHOR NOTE}

The author thanks William Batchelder, Henrik Olsson, and Thomas Wallsten for their insightful comments on this article. Any mistakes that remain are the sole responsibility of the author. Correspondence concerning this article should be sent to E. C. Merkle, Department of Psychology, Wichita State University, 1845 Fairmount Box 34, Wichita, KS 67260 (e-mail: edgar.merkle@wichita.edu).

\section{REFERENCES}

Arkes, H. R., Christensen, C., LaI, C., \& Blumer, C. (1987). Two methods of reducing overconfidence. Organizational Behavior \& Human Decision Processes, 39, 133-144.

BJöRKMAN, M. (1994). Internal cue theory: Calibration and resolution of confidence in general knowledge. Organizational Behavior \& Human Decision Processes, 58, 386-405.

Casella, G., \& Berger, R. L. (2002). Statistical inference (2nd ed.). Pacific Grove, CA: Duxbury Press.

DAWES, R. M. (1980). Confidence in intellectual vs. confidence in perceptual judgments. In E. D. Lantermann \& H. Feger (Eds.), Similarity and choice: Papers in honor of Clyde Coombs (pp. 327-345). Bern: Han Huber.

Dawes, R. M., \& Mulford, M. (1996). The false consensus effect and overconfidence: Flaws in judgment or flaws in how we study judgment? Organizational Behavior \& Human Decision Processes, 65, 201-211.

DougherTy, M. R. P. (2001). Integration of the ecological and error models of overconfidence using a multiple-trace memory model. Journal of Experimental Psychology: General, 130, 579-599.

Erev, I., Wallsten, T. S., \& Budescu, D. V. (1994). Simultaneous over- and underconfidence: The role of error in judgment processes. Psychological Review, 101, 519-527.

Ferrell, W. R., \& McGoey, P. J. (1980). A model of calibration for subjective probabilities. Organizational Behavior \& Human Decision Processes, 26, 32-53.

Gigerenzer, G., Hoffrage, U., \& Kleinbölting, H. (1991). Probabilistic mental models: A Brunswikian theory of confidence. Psychological Review, 98, 506-528.

Griffin, D., \& TVersky, A. (1992). The weighing of evidence and the determinants of confidence. Cognitive Psychology, 24, 411-435.

Juslin, P., Olsson, H., \& Buörkman, M. (1997). Brunswikian and Thurstonian origins of bias in probability assessment: On the interpretation of stochastic components of judgment. Journal of Behavioral Decision Making, 10, 189-209.

Juslin, P., Winman, A., \& Olsson, H. (2000). Naive empiricism and dogmatism in confidence research: A critical examination of the hard-easy effect. Psychological Review, 107, 384-396.

Juslin, P., Winman, A., \& Olsson, H. (2003). Calibration, additivity, and source independence of probability judgments in general knowledge and sensory discrimination tasks. Organizational Behavior \& Human Decision Processes, 92, 34-51.

KeREN, G. (1988). On the ability of monitoring non-veridical perceptions and uncertain knowledge: Some calibration studies. Acta Psychologica, 67, 95-119.

Klayman, J., Soll, J. B., González-Vallejo, C., \& Barlas, S. (1999). Overconfidence: It depends on how, what, and whom you ask. Organizational Behavior and Human Decision Processes, 79, 216-247.

Lichtenstein, S., \& FischHOFF, B. (1977). Do those who know more also know more about how much they know? The calibration of probability judgments. Organizational Behavior \& Human Performance, 20, $159-183$

Lichtenstein, S., Fischhoff, B., \& Phillips, L. D. (1982). Calibration of probabilities: The state of the art to 1980. In D. Kahneman, P. Slovic, \& A. Tversky (Eds.), Judgment under uncertainty: Heuristics and biases (pp. 306-334). Cambridge: Cambridge University Press.

Lindsay, D. S., Nilsen, E., \& READ, J. D. (2000). Witnessing-condition heterogeneity and witnesses' versus investigators' confidence in the accuracy of witnesses' identification decisions. Law \& Human Behavior, 24, 685-697.

McKenZIE, C. R. M. (1997). Underweighting alternatives and overconfidence. Organizational Behavior \& Human Decision Processes, 71, 141-160.

Merkle, E. C., Sieck, W. R., \& VAN Zandt, T. (2008). Response error and processing biases in confidence judgment. Journal of Behavioral Decision Making, 21, 428-448.

Merkle, E. C., \& VAn Zandt, T. (2006). An application of the Poisson race model to confidence calibration. Journal of Experimental Psychology: General, 135, 391-408.

Mueller, S. T., \& Weidemann, C. T. (2008). Decision noise: An explanation for observed violations of signal detection theory. Psychonomic Bulletin \& Review, 15, 465-494.

Murphy, A. H., \& WinKLeR, R. L. (1984). Probability forecasting in meteorology. Journal of the American Statistical Association, 79, 489-500. MyUnG, I. J., \& PitT, M. A. (2001). Mathematical modeling. In H. Pash- 
ler (Series Ed.) \& J. Wixted (Vol. Ed.), Stevens' Handbook of experimental psychology (3rd ed., Vol. 4, pp. 429-460). New York: Wiley.

O'Hagan, A., Buck, C. E., Daneshrhah, A., Eiser, J. R., GarthWAITE, P. H., JENKINSON, D. J., ET AL. (2006). Uncertain judgements: Eliciting experts' probabilities. Hoboken: Wiley.

Olsson, H., Juslin, P., \& Winman, A. (2008). Comments: The role of random error in confidence judgment: Reply to Merkle, Sieck, and Van Zandt. Journal of Behavioral Decision Making, 21, 449-452.

PRICE, P. C. (1998). Effects of a relative-frequency elicitation question on likelihood judgment accuracy: The case of external correspondence. Organizational Behavior \& Human Decision Processes, 76, 277-297.

RoNis, D. L., \& Yates, J. F. (1987). Components of probability judgment accuracy: Individual consistency and effects of subject matter and assessment method. Organizational Behavior \& Human Decision Processes, 40, 193-218.

Sieck, W. R., Merkle, E. C., \& Van Zandt, T. (2007). Option fixation: A cognitive contributor to overconfidence. Organizational Behavior \& Human Decision Processes, 103, 68-83.

Suantak, L., Bolger, F., \& Ferrell, W. R. (1996). The hard-easy effect in subjective probability calibration. Organizational Behavior \& Human Decision Processes, 67, 201-221.

Thomson, M. E., Önkal-Atay, D., Pollock, A. C., \& Macaulay, A. (2003). The influence of trend strength on directional probabilistic currency predictions. International Journal of Forecasting, 19, 241-256.

VON WINTERFELDT, D., \& EDWARDS, W. (1986). Decision analysis and behavioral research. New York: Cambridge University Press.

WaLLSTEN, T. S. (1996). An analysis of judgment research analyses. Organizational Behavior \& Human Decision Processes, 65, 220-226.

Weber, N., \& Brewer, N. (2004). Confidence-accuracy calibration in absolute and relative face recognition judgments. Journal of Experimental Psychology: Applied, 10, 156-172.

Wells, G. L., Ferguson, T. J., \& Lindsay, R. C. L. (1981). The tractability of eyewitness confidence and its implications for triers of fact. Journal of Applied Psychology, 66, 688-696.

\section{NOTES}

1. This task is commonly employed by decision researchers (see, e.g., Arkes, Christensen, Lai, \& Blumer, 1987; Dawes, 1980; Gigerenzer et al., 1991; Lichtenstein \& Fischhoff, 1977).

2. Juslin, Olsson, and Björkman (1997) referred to this model as the response error model. I generically call it an error model here, because the phrase response error is ill defined and could lead readers to believe that the error term only captures one type of error. For differing definitions of response error, see Merkle et al. (2008) and Olsson et al. (2008).

3 . The unbounded error allows for the possibility that $f_{j k}$ exceeds its bounds. As stated in the Discussion section and shown in Appendix B, this has no effect on the derivations in this article.

4. Showing this involves expressing $E[t(1-t)]$ as $E(t)-E\left(t^{2}\right)$. For $0 \leq t \leq 1, E(t) \geq E\left(t^{2}\right)$ with equality holding only when $P(t=0)+$ $P(t=1)=1$. In other words, $E[t(1-t)]=0$ only when the judge is certain of every item on a test.

5. Juslin et al. (2000) described this type of miscalibration as "linear dependency," though they did not explicitly relate it to perfectly calibrated judges. Furthermore, Klayman, Soll, González-Vallejo, and Barlas (1999) presented a method for removing linear dependency from observed confidence data.

6 . The .167 figure is an absolute lowest bound for observing a hardeasy effect. If we take into account $\operatorname{corr}(\bar{f}, \bar{d})$ (see Proposition 1A), then we can still observe a hard-easy effect when $\sigma^{2}$ is greater than .167 .

7. This statement may appear to conflict with my analyses in this article, wherein I have assumed a single model of confidence and choice. The distinction between a model's ability to fit data versus its ability to describe the confidence elicitation process becomes important here: I assume that the error model can sufficiently fit observed data even if its explanation of the confidence elicitation process is incorrect. Thus, the true confidence elicitation process can involve separate mechanisms for confidence and choice, while still yielding calibration data similar to those of the model used in this article.

\section{APPENDIX A}

\section{Derivation of $\operatorname{var}(\bar{d})$ and $\operatorname{var}(\bar{f})$ for the Error Model}

In this appendix, I derive $\operatorname{var}(\bar{d})$ and $\operatorname{var}(\bar{f})$ (Equations 4 and 5) under the error model used in this article. I first present a general version of the model, and I then discuss the specific assumptions employed for the derivations.

Let $d_{j k}$ consist of exchangeable $0 / 1$ variables denoting whether or not a judge correctly answers item $k$ $(k=1, \ldots, K)$ on test $j(j=1, \ldots, J)$. Each $d_{j k}$ depends on a latent variable $t_{j k}$ in $(0,1)$ with distribution $g_{j}(t)$, such that $f\left(d_{j k} \mid t_{j k}\right) \sim \operatorname{Bernoulli}\left(t_{j k}\right)$.

The $f_{j k}$ values depend on $t_{j k}$ such that

$$
f_{j k}=t_{j k}+e_{j k}
$$

where the $\mathrm{e}_{j k}$ values are independent and follow a distribution $h_{j}(e)$ with mean 0 . Independent sampling of $t_{j k}$ from $g_{j}(t)$ implies that the $d_{j k}$ values are independent of one another (and also that the $f_{i j}$ values are independent of one another).

In the error model described in this article, I assumed specific distributions for the model above. First, I assumed that $g_{j}(t) \sim \operatorname{Beta}(\alpha, \beta) \forall j$. In other words, in the model, it is assumed that item difficulty follows the same distribution from test to test. This assumption is similar to those behind experiments that randomly sample test items from a specific domain (e.g., Gigerenzer et al., 1991). Next, the model assumes that $h_{j}(e) \sim \mathrm{N}\left(0, \sigma^{2}\right) \forall j$. This implies that the judge's response error distribution is constant across items and tests. Although these assumptions simplify the derivations, they are not necessary to demonstrate the ubiquity of the hard-easy effect.

Employing the assumptions above, we can obtain the unconditional variance of $\bar{d}$ via the conditional variance identity (see, e.g., Casella \& Berger, 2002):

$$
\begin{aligned}
\operatorname{var}(\bar{d}) & =\operatorname{var}\left(\frac{1}{J} \sum_{j} \frac{1}{K} \sum_{k} d_{j k}\right) \\
& =\frac{1}{(J K)^{2}} \sum_{j} \sum_{k}\left\{E\left[\operatorname{var}\left(d_{j k} \mid t_{j k}\right)\right]+\operatorname{var}\left[E\left(d_{j k} \mid t_{j k}\right)\right]\right\} \\
& =\frac{1}{(J K)^{2}} \sum_{j} \sum_{k}\left\{E\left[t_{j k}\left(1-t_{j k}\right)\right]+\operatorname{var}\left(t_{j k}\right)\right\} \\
& =\frac{1}{J K}\{E[t(1-t)]+\operatorname{var}(t)\} .
\end{aligned}
$$




\section{APPENDIXA (Continued)}

The variance of $\bar{f}$ is

$$
\begin{aligned}
\operatorname{var}(\bar{f}) & =\operatorname{var}\left(\frac{1}{J} \sum_{j} \frac{1}{K} \sum_{k} f_{j k}\right) \\
& =\frac{1}{(J K)^{2}} \sum_{j} \sum_{k}\left[\operatorname{var}\left(t_{j k}\right)+\operatorname{var}\left(e_{j k}\right)\right] \\
& =\frac{1}{J K}\left[\operatorname{var}(t)+\sigma^{2}\right] .
\end{aligned}
$$

When $\sigma^{2}<E[t(1-t)], \operatorname{var}(\bar{f})<\operatorname{var}(\bar{d})$. This implies a hard-easy effect (see Proposition 1$)$.

\section{APPENDIX B}

Bounded Error

In this appendix, I show that some of the results in the article hold when accounting for the $(0,1)$ bounds of the confidence scale. Following Erev et al. (1994), error is added to the unbounded log odds of $t_{j k}$ to yield an intermediate variable $x_{j k}$ :

$$
x_{j k}=\log \left(\frac{t_{j k}}{1-t_{j k}}\right)+e_{j k},
$$

where $e_{j k} \sim \mathrm{N}\left(0, \sigma^{2}\right)$. The intermediate variable, $x_{j k}$, is then transformed back to the $(0,1)$ scale to yield $f_{j k}$ :

$$
f_{j k}=\frac{\exp \left(x_{j k}\right)}{1+\exp \left(x_{j k}\right)} .
$$

Closed-form expressions for $\operatorname{var}(\bar{f})$ are unavailable, so I conducted a simulation study to examine the situations under which a hard-easy effect is observed.

For the simulations, I allowed the two test difficulty parameters (Beta distribution parameters $\alpha$ and $\beta$ ) to vary from 0.001 to 30.001 in increments of 0.5 . This specific range was chosen on the basis of the analyses in the main text, which show that the hard-easy effect disappears only at small values of $\alpha$ and $\beta$. Furthermore, I allowed the error variance parameter $\left(\sigma^{2}\right)$ to vary from 0.001 to 1.9 in increments of 0.02 . The $0.001-1.9 \sigma^{2}$ range on the log-odds scale approximates the $0-0.03 \sigma^{2}$ range on the untransformed confidence scale.

For each combination of parameter values, I generated 1,000 tests of 50 items each. In calculating $\bar{d}$ and $\bar{f}$ for each test, the presence of a hard-easy effect was assessed by examining whether $\operatorname{cov}(\bar{d}, \mathrm{OC})<0$. Of the 353,495 data sets generated, hard-easy effects were observed in all but 146 . For all 146 data sets in which the hard-easy effect was absent, either $\alpha$ or $\beta$ equaled 0.001 . These findings support the claim that the hard-easy effect appears in all realistic situations.

(Manuscript received February 25, 2008;

revision accepted for publication September 5, 2008.) 